\title{
Antioxidant and Antimicrobial Effects of Lemongrass (Cymbopogon citrates) Oil on the Quality Characteristics of Camel Burger "Camburger" under Refrigerated Storage
}

\author{
Engy F. Zaki ${ }^{1}$, Abdel Aziz Nadir ${ }^{2 *}$, Ibrahim Mohamed Fouad Helmy ${ }^{2}$ and \\ Nahed M. Abdel Maguid ${ }^{2}$
}

${ }^{I}$ Department of Animal Breeding, Meat Production and Technology Unit, Desert Research Center, 11753 Cairo, Egypt

${ }^{2}$ Department of food science, National Research Center, Dokki, Cairo, Egypt

*Corresponding author

\begin{abstract}
A B S T R A C T
Keywords

Camel burger, Lemongrass oil, Quality characteristics, Cold storage

Article Info

Accepted: 28 February 2018 Available Online: 10 March 2018

This study evaluated the use of lemongrass oil $(0.5,0.75$ and $1 \%)$ in the formulation of camel burger on some chemical, physical, microbiological and sensory characteristic during cold storage at $4^{\circ} \mathrm{C}$ for 12 days. Increased the level of lemongrass improved the physical properties of camel burger. The lowest cooking loss and reduction in diameter \% was found in camel burger formulated with lemongrass oil. During cold storage TBA and TVBN values were significantly increased as the time of storage increased but camel burger formulated with lemongrass oil had the lowest values than control sample. Cambuger formulated with $1 \%$ lemongrass showed the lowest total bacterial count than the other samples. Sensory evaluation of camel burger samples showed that camburger formulated with $1 \%$ lemongrass recorded the highest score for aroma, taste, color, texture and overall acceptability while, the control burger had the lowest score.
\end{abstract}

\section{Introduction}

Lipid oxidation and growth of undesirable microorganisms in food products result in the development of spoilage, rancidity and deterioration, rendering such products unacceptable for human consumption (Bozin et al., 2007). In an effort to retard this process, several synthetic food additives have been widely used in the meat industry. However, the application of synthetic antioxidants has been recently restricted due to the toxicological effects of synthetic antioxidants and there is suspicion that they are carcinogenic. Consumers have shown preference for the use of natural antioxidants (Byrd, 2001; Johnston et al., 2005). Natural plants can inhibit oxidative rancidity and delay the development of off-flavor in some products.

Lemongrass (Cymbopogon citratus), a tall perennial grass comprising of about 55 species, is native to warm region and grows in 
almost all tropical and subtropical countries (Cheel et al., 2005). It is an aromatic herb, known in the North and West tropical Africa, in Arabian Peninsula and in Egypt (Khadri et al., 2010). Due to the production of lemongrass oil as major component, two of the species i.e. Cymbopogan citrates and C. flexuosus are generally called Lemongrass.

Therefore, the aim of this study to investigate the effect of added different levels of lemongrass oil as antioxidant and antimicrobial agent on the chemical, physical, microbial characteristics and sensory evaluation of camel burger during cold storage.

\section{Materials and Methods}

The present work was carried out at Desert Research Center (DRC), El Matarya, Cairo, Egypt, and National Research Center (NRC) Dokki, Cairo, Egypt.

\section{Plant material}

Fresh plant materials of Lemongrass (Cymbopogon citrates) were obtained from Siwa Research Station belonging Desert Research Center, Egypt.

\section{Preparation of lemongrass oil}

Fresh plant materials of lemongrass (Cymbopogon citrates) were subject to hydrodistillation using a fabricated distillation apparatus as described by Akin-Osanaiye et al., (2007).

\section{Gas Chromatography-mass Spectrometry (GC-MS) Analysis}

The analysis performed using Perkin Elmer Auto system equipped with a capillary column BPX5 (30 m x $0.25 \mathrm{~mm}$ i.d. and $0.25 \mathrm{~mm} \mu \mathrm{m}$ film thickness) was used for separation of the component. Essential oils were positively identified by matching their RI values and mass spectra with those of standards, also run under identical chromatographic conditions in the laboratory (Adams, 2007).

\section{Preparation of camel burger (Camburger)}

Camel meat and humped fat obtained from local slaughter house were used in this study. Left round of 3-4 years aged camel were pooled to form an experiment unit, with three (batches) of lean ground meat being prepared from each burger formulation. Lean meat was ground through a $3 \mathrm{~mm}$ plate grinder.

The ingredients and lemongrass oil $(0.5,0.75$ and $1 \%$ ) were added as given in Table 1. Each formula was handily mixed and formed by using manual burger press machine.

Burgers were placed in plastic foam meat trays, packed in polyethylene bags and stored at $4 \pm 1^{\circ} \mathrm{C}$ for 12 days until analysis.

\section{Chemical analysis}

The extent of lipid oxidation in camel burger was assessed by measuring 2- thiobarbituric acid reactive substances (TBARS), as described by Rosmini et al., (1996)

Total volatile basic nitrogen (TVBN) of camel burger was determined according to Kirk and Sawyer (1991).

\section{Physical analysis}

$\mathrm{pH}$ of raw camel burger was measured using a digital pH-meter (Jenway 3510 conductivity and $\mathrm{pH}$ meter) as described by Defreitas et al., (1997).

Water holding capacity (W.H.C) and plasticity of samples were measured using the method of Wierbicki and Deatherage (1958). 


\section{Cooking measurements}

All cooking measurements were carried out on three replicates of each treatment as reported by AMSA (1995).

(Uncooked sample weight) (Cooked sample weight) $\times 100$

Cooking loss $(\%)=$

(Uncooked sample weight)

Raw and cooked samples were measured for diameter of burger as described by Berry (1993) using the following equation:

(Uncooked sample diameter) (Cooked sample diameter) $\times 100$ Reduction in diameter $(\%)=$

(Uncooked sample diameter)

\section{Sensory evaluation}

Camel burger was subjected to organoleptic evaluation as described by Mailgaad et al., (1999).

\section{Microbiology analysis}

Duplicated 10g each of camel burger samples were collected aseptically immediately after each storage period and diluted in $90 \mathrm{~mL}$ of tryptone salt solution $(0.3 \%$ tryptone and $\mathrm{NaCl}$ at $0.85 \%$, sterilized at $121^{\circ} \mathrm{C}$ for $15 \mathrm{~min}$ ) and homogenized for $30 \mathrm{~s}$.

Successive decimal dilutions were performed in test tubes containing $9 \mathrm{~mL}$ sterile tryptone salt for analyses of total bacterial count, and total psychrophilic according to the microorganisms appropriate for meat as recommended by Villa et al., (2016).

Total bacterial counts determined as described by (ISO4833, 1991). The plates are incubated for 72 hours at $30^{\circ} \mathrm{C}$. Psychrophilic counts were determined by the technique described by (Portuguese Standard2307, 1987). The plates are incubated at $7^{\circ} \mathrm{C} \pm 1$ for 10 days.

\section{Statistical analysis}

One - way ANOVA was applied for sensory evaluation. The effects of addition of lemongrass oil and storage time were subject to two - way ANOVA. The results were expressed as mean $\pm \mathrm{SE}$, according to Armitage and Berry (1987).

\section{Results and Discussion}

\section{Chemical composition of lemongrass oil}

The chemical composition of lemongrass oil are presented in Table 2, it can be found that the oil consist of monoterpene fraction. This monoterpene fraction mainly composed of a high percentage of geranial (44.76), neral (30.87), myrecene (7.59). Citral is a natural mixture of geranial and neral. These results are close to that obtained by Matasyoh et al., (2011) they found the essential oil of $C$. citrates (Lemongrass) dominated by monoterpene hydrocarbons which accounted for $94.25 \%$ of the total oil and characterized by a high percentage of geranial $(39.53 \%)$, neral $(33.31 \%)$, and myrecene $(11.41 \%)$. The same results were found by Barbosa et al., (2008).

The physical properties of camel burger during cold storage are shown in Table 3 . Control camburger had the highest cooking loss $\%$. During cold storage the cooking loss was significantly decreased after 3 day of storage for all camburger samples, but no significant differences were found during the rest of storage period.

Control camburger had the highest reduction in diameter percentage than the formulated samples. No significant differences were found between camburger formulated with 0.5 
and /or $0.75 \%$ lemongrass oil, the lowest percentage was found in camburger formulated with $1 \%$ lemongrass. During cold storage reduction in diameter \% was increased with increase of cold storage for all camburger. These results are close to that obtained by Hussein et al., (2015).

WHC and plasticity of all camel burger samples were significantly increased as the time of cold storage increased. At any time of cold storage camel burger formulated with lemongrass oil showed the highest value than control samples. Generally, addition of lemongrass oil in the formulation of camel burger improved the physical properties of the product during cold storage at $4^{\circ} \mathrm{C}$ for 12 days. The TVBN value of control and formulated camburger during cold storage are presented in Table 4 . The control sample had the higher TVBN than other camburger containing different levels of lemongrass. TVBN value decreased as the level of lemongrass increased. During cold storage TVBN values were significantly increased as the time of storage increased in both control and formulated camburger. These results are agreed with Ibrahim and Abu Salem (2013). This increase of VBN in patties sample during cold storage might be attributed to the breakdown protein as a result of activity of microbial strains and proteolysis enzymes.

Table.1 Camburger formulated with different levels of lemongrass oil

\begin{tabular}{|c|c|c|c|c|}
\hline $\begin{array}{c}\text { Ingredients } \\
(\%)\end{array}$ & Control & Formula (1) & Formula (2) & Formula (3) \\
\hline Camel meat & 67.0 & 67.0 & 67.0 & 67.0 \\
\hline Camel fat & 8.0 & 8.0 & 8.0 & 8.0 \\
\hline Lemongrass oil & ---- & 0.5 & 0.75 & 1.0 \\
\hline Onion & 7.0 & 7.0 & 7.0 & 7.0 \\
\hline Salt & 2.0 & 2.0 & 2.0 & 2.0 \\
\hline Rusk & 5.0 & 5.0 & 5.0 & 5.0 \\
\hline Spices & 1.0 & 1.0 & 1.0 & 1.0 \\
\hline Ice water & 10.0 & 10.0 & 10.0 & 10.0 \\
\hline
\end{tabular}

Table.2 Chemical composition of lemongrass oil

\begin{tabular}{|c|c|c|c|c|}
\hline Serial number & Compound & R.I. & $\begin{array}{c}\text { Oil chemical } \\
\text { composition \% }\end{array}$ & $\begin{array}{c}\text { Molecular } \\
\text { formula }\end{array}$ \\
\hline $\mathbf{1}$ & B-Myercene & 958 & 7.59 & $\mathrm{C}_{10} \mathrm{H}_{16}$ \\
\hline 2 & Ocimene & 958 & 0.21 & $\mathrm{C}_{10} \mathrm{H}_{16}$ \\
\hline $\mathbf{3}$ & B-Ocimene & 976 & 0.58 & $\mathrm{C}_{10} \mathrm{H}_{16}$ \\
\hline $\mathbf{4}$ & Linalool & 1082 & 0.56 & $\mathrm{C}_{10} \mathrm{H}_{18} \mathrm{O}$ \\
\hline $\mathbf{5}$ & 3-Undcyne & 1132 & 1.98 & $\mathrm{C}_{11} \mathrm{H}_{20}$ \\
\hline $\mathbf{6}$ & Neral & 1174 & 30.87 & $\mathrm{C}_{10} \mathrm{H}_{16} \mathrm{O}$ \\
\hline 7 & Geranial & 1174 & 44.76 & $\mathrm{C}_{10} \mathrm{H}_{16} \mathrm{O}$ \\
\hline $\mathbf{8}$ & Citronllol & 1179 & 0.32 & $\mathrm{C}_{10} \mathrm{H}_{20} \mathrm{O}$ \\
\hline $\mathbf{9}$ & Nerol & 1228 & 3.39 & $\mathrm{C}_{10} \mathrm{H}_{18} \mathrm{O}$ \\
\hline $\mathbf{1 0}$ & $\boldsymbol{\alpha - F a r n e s e n e}$ & 1458 & 0.24 & $\mathrm{C}_{15} \mathrm{H}_{24}$ \\
\hline $\mathbf{1 1}$ & 8-Cadinene & 1469 & 0.15 & $\mathrm{C}_{15} \mathrm{H}_{24}$ \\
\hline
\end{tabular}


Table.3 Physical properties of camburger formulated with lemongrass oil and storage at $4 \pm 1^{\circ} \mathrm{C}$ for 12 days

\begin{tabular}{|c|c|c|c|c|c|}
\hline \multirow[t]{2}{*}{ Treatments } & \multicolumn{5}{|c|}{ Storage period (days) } \\
\hline & $\mathbf{0}$ & 3 & 6 & 9 & 12 \\
\hline \multicolumn{6}{|c|}{ Plasticity $\left(\mathrm{cm}^{2}\right)$} \\
\hline Control & $\begin{array}{c}2.86^{\mathrm{Ca}} \pm 0 \\
.11\end{array}$ & $\begin{array}{l}2.78^{\mathrm{Ca}} \\
\pm 0.13\end{array}$ & $\begin{array}{l}2.69^{\mathrm{Ca}} \\
\pm 0.17\end{array}$ & $\begin{array}{l}2.57^{\mathrm{Cb}} \\
\pm 0.19\end{array}$ & $\begin{array}{l}2.46^{\mathrm{Cc}} \\
\pm 0.15\end{array}$ \\
\hline Formula (1) & $\begin{array}{c}2.93^{\mathrm{Ba}} \pm 0 \\
.15\end{array}$ & $\begin{array}{l}2.85^{\mathrm{Ba}} \\
\pm 0.18\end{array}$ & $\begin{array}{l}2.76^{\mathrm{Ba}} \\
\pm 0.20\end{array}$ & $\begin{array}{l}2.65^{\mathrm{Bb}} \\
\pm 0.13\end{array}$ & $\begin{array}{c}2.56^{\mathrm{Bb}} \pm 0 \\
.10\end{array}$ \\
\hline Formula (2) & $\begin{array}{c}3.02^{\mathrm{Aa}} \pm 0 \\
.16\end{array}$ & $\begin{array}{l}3.11^{\mathrm{Aa}} \\
\pm 0.24\end{array}$ & $\begin{array}{l}3.20^{\mathrm{Aa}} \\
\pm 0.08\end{array}$ & $\begin{array}{l}3.29^{\mathrm{Aa}} \\
\pm 0.15\end{array}$ & $\begin{array}{l}3.36^{\text {Aa }} \\
\pm 0.30\end{array}$ \\
\hline Formula (3) & $\begin{array}{c}3.10^{\mathrm{Ac}} \pm 0 \\
.14\end{array}$ & $\begin{array}{l}3.21^{\mathrm{Ab}} \\
\pm 0.12\end{array}$ & $\begin{array}{l}3.30^{\mathrm{Ab}} \\
\pm 0.34\end{array}$ & $\begin{array}{l}3.37^{\mathrm{Aa}} \\
\pm 0.16\end{array}$ & $\begin{array}{l}3.41^{\mathrm{Aa}} \\
\pm 0.12\end{array}$ \\
\hline \multicolumn{6}{|c|}{ WHC $\left(\mathrm{cm}^{2}\right)$} \\
\hline Control & $\begin{array}{c}3.40^{\mathrm{Bd}} \pm 0 \\
.21\end{array}$ & $\begin{array}{l}3.52^{\mathrm{Bc}} \\
\pm 0.26\end{array}$ & $\begin{array}{l}3.63^{\mathrm{Bb}} \\
\pm 0.24\end{array}$ & $\begin{array}{l}3.90^{\mathrm{Ba}} \\
\pm 0.17\end{array}$ & $\begin{array}{l}3.81^{\mathrm{Ba}} \\
\pm 0.29\end{array}$ \\
\hline Formula (1) & $\begin{array}{c}3.44^{\mathrm{Bd}} \pm 0 \\
.30\end{array}$ & $\begin{array}{l}3.58^{\mathrm{Bc}} \\
\pm 0.07\end{array}$ & $\begin{array}{l}3.72^{\mathrm{Bb}} \\
\pm 0.16\end{array}$ & $\begin{array}{l}3.80^{\mathrm{Ba}} \\
\pm 0.23\end{array}$ & $\begin{array}{l}3.88^{\mathrm{Ba}} \\
\pm 0.25\end{array}$ \\
\hline Formula (2) & $\begin{array}{c}3.50^{\mathrm{Ad}} \pm 0 \\
.20\end{array}$ & $\begin{array}{l}3.67^{\mathrm{Ac}} \\
\pm 0.31\end{array}$ & $\begin{array}{l}3.81^{\mathrm{Ab}} \\
\pm 0.37\end{array}$ & $\begin{array}{l}3.90^{\mathrm{Aa}} \\
\pm 0.16\end{array}$ & $\begin{array}{l}3.98^{\mathrm{Aa}} \\
\pm 0.14\end{array}$ \\
\hline Formula (3) & $\begin{array}{c}3.58^{\mathrm{Ad}} \pm 0 \\
.30\end{array}$ & $\begin{array}{l}3.71^{\mathrm{Ac}} \\
\pm 0.34\end{array}$ & $\begin{array}{l}3.88^{\mathrm{Ab}} \\
\pm 0.30\end{array}$ & $\begin{array}{l}3.97^{\mathrm{Aa}} \\
\pm 0.25\end{array}$ & $\begin{array}{l}4.07^{\mathrm{Aa}} \\
\pm 0.28\end{array}$ \\
\hline \multicolumn{6}{|c|}{ Reduction in diameter $(\%)$} \\
\hline Control & $\begin{array}{c}17.64^{\mathrm{Ac}} \pm 0 \\
.13\end{array}$ & $\begin{array}{c}17.82^{\mathrm{Ab}} \pm 0 \\
.09\end{array}$ & $\begin{array}{c}17.95^{\mathrm{Ab}} \pm 0 \\
.11\end{array}$ & $\begin{array}{c}18.06^{\mathrm{Aa}} \\
\pm 0.12\end{array}$ & $\begin{array}{c}18.19^{\text {Aa }} \\
\pm 0.14\end{array}$ \\
\hline Formula (1) & $\begin{array}{c}16.59^{\mathrm{Bc}} \pm 0 \\
.14\end{array}$ & $\begin{array}{c}16.71^{\mathrm{Bb}} \pm 0 \\
.23\end{array}$ & $\begin{array}{c}16.84^{\mathrm{Bb}} \pm 0 \\
.27\end{array}$ & $\begin{array}{c}16.98^{\mathrm{Ba}} \\
\pm 0.29\end{array}$ & $\begin{array}{c}17.05^{\mathrm{Ba}} \\
\pm 0.19\end{array}$ \\
\hline Formula (2) & $\begin{array}{c}16.48^{\mathrm{Bc}} \pm 0 \\
.34\end{array}$ & $\begin{array}{c}16.64^{\mathrm{Bb}} \pm 0 \\
.21\end{array}$ & $\begin{array}{c}16.75^{\mathrm{Bb}} \pm 0 \\
.23\end{array}$ & $\begin{array}{c}16.87^{\mathrm{Ba}} \\
\pm 0.18\end{array}$ & $\begin{array}{c}16.95^{\mathrm{Ba}} \\
\pm 0.09\end{array}$ \\
\hline Formula (3) & $\begin{array}{c}16.29^{\mathrm{Cc}} \pm 0 \\
.17\end{array}$ & $\begin{array}{c}16.43^{\mathrm{Cb}} \pm 0 \\
.11\end{array}$ & $\begin{array}{c}16.60^{\mathrm{Cb}} \pm 0 \\
.19\end{array}$ & $\begin{array}{c}16.71^{\mathrm{Ca}} \\
\pm 0.37\end{array}$ & $\begin{array}{c}16.80^{\mathrm{Ca}} \\
\pm 0.32\end{array}$ \\
\hline \multicolumn{6}{|c|}{ Cooking loss (\%) } \\
\hline Control & $\begin{array}{c}24.76^{\mathrm{Ac}} \pm 0 \\
.20\end{array}$ & $\begin{array}{c}24.90^{\mathrm{Ab}} \pm 0 \\
.17\end{array}$ & $\begin{array}{c}25.03^{\mathrm{Aa}} \pm 0 \\
10\end{array}$ & $\begin{array}{c}25.12^{\mathrm{Aa}} \\
\pm 0.23\end{array}$ & $\begin{array}{c}25.21^{\mathrm{Aa}} \\
\pm 0.28\end{array}$ \\
\hline Formula (1) & $\begin{array}{c}23.51^{\mathrm{Bc}} \pm 0 \\
.10\end{array}$ & $\begin{array}{c}23.70^{\mathrm{Bb}} \pm 0 \\
.34\end{array}$ & $\begin{array}{c}23.83^{\mathrm{Ba}} \pm 0 . \\
29\end{array}$ & $\begin{array}{c}23.95^{\mathrm{Ba}} \\
\pm 0.37\end{array}$ & $\begin{array}{c}24.02^{\mathrm{Ba}} \\
\pm 0.18\end{array}$ \\
\hline Formula (2) & $\begin{array}{c}23.39^{\mathrm{Bc}} \pm 0 \\
.27\end{array}$ & $\begin{array}{c}23.58^{\mathrm{Bb}} \pm 0 \\
.19\end{array}$ & $\begin{array}{c}23.76^{\mathrm{Ba}} \\
\pm 0.16\end{array}$ & $\begin{array}{c}23.84^{\mathrm{Ba}} \\
\pm 0.12\end{array}$ & $\begin{array}{c}23.96^{\mathrm{Ba}} \\
\pm 0.07\end{array}$ \\
\hline Formula (3) & $\begin{array}{c}22.28^{\mathrm{Bc}} \pm 0 \\
.19\end{array}$ & $\begin{array}{c}22.46^{\mathrm{Cb}} \pm 0 \\
.23\end{array}$ & $\begin{array}{c}22.64^{\mathrm{Ca}} \\
\pm 0.14\end{array}$ & $\begin{array}{c}22.73^{\mathrm{Ca}} \\
\pm 0.08\end{array}$ & $\begin{array}{c}22.81^{\mathrm{Ca}} \\
\pm 0.13\end{array}$ \\
\hline
\end{tabular}

${ }^{A-C}$ means with different superscripts within the same column for each parameter are significantly different.

${ }^{a-c}$ different superscripts in the same row are significantly $(\mathrm{P}<0.05)$ different. 
Table.4 Chemical properties of camburger formulated with lemongrass oil and storage at $4 \pm 1^{\circ} \mathrm{C}$ for 12 days

\begin{tabular}{|c|c|c|c|c|c|}
\hline \multicolumn{6}{|c|}{ Storage period (days) } \\
\hline samples & $\mathbf{0}$ & 3 & 6 & 9 & 12 \\
\hline & \multicolumn{5}{|c|}{ TVBN(mg/kg) } \\
\hline Control & $4.20^{\mathrm{Ad}} \pm 0.23$ & $5.24^{\mathrm{Acd}} \pm 0.12$ & $6.30^{\mathrm{Abc}} \pm 0.16$ & $7.37^{\mathrm{Aab}} \pm 0.19$ & $8.42^{\mathrm{Aa}} \pm 0.23$ \\
\hline Formula (1) & $3.86^{\mathrm{Bd}} \pm 0.29$ & $4.69^{\mathrm{Bcd}} \pm 0.32$ & $5.51^{\mathrm{Bbc}} \pm 0.22$ & $6.40^{\mathrm{Bab}} \pm 0.15$ & $7.32^{\mathrm{Ba}} \pm 0.17$ \\
\hline Formula (2) & $3.28^{\mathrm{Bd}} \pm 0.13$ & $4.15^{\mathrm{Bcd}} \pm 0.39$ & $5.20^{\mathrm{Bbc}} \pm 0.36$ & $6.07^{\mathrm{Bab}} \pm 0.24$ & $6.98^{\mathrm{Ba}} \pm 0.14$ \\
\hline \multirow[t]{2}{*}{ Formula (3) } & $3.00^{\mathrm{Bd}} \pm 0.16$ & $3.90^{\mathrm{Bcd}} \pm 0.32$ & $4.86^{\mathrm{Bbc}} \pm 0.18$ & $5.91^{\mathrm{Bab}} \pm 0.17$ & $6.75^{\mathrm{Ba}} \pm 0.20$ \\
\hline & \multicolumn{5}{|c|}{ TBA (mg/kg) } \\
\hline Control & $0.57^{\mathrm{Ac}} \pm 0.36$ & $0.59^{\mathrm{Abc}} \pm 0.10$ & $0.61^{\mathrm{Ab}} \pm 0.17$ & $0.63^{\mathrm{Aab}} \pm 0.11$ & $0.65^{\mathrm{Aa}} \pm 0.13$ \\
\hline Formula (1) & $0.52^{\mathrm{Bc}} \pm 0.18$ & $0.55^{\mathrm{Bbc}} \pm 0.20$ & $0.58^{\mathrm{Bb}} \pm 0.27$ & $0.60^{\mathrm{Bab}} \pm 0.21$ & $0.62^{\mathrm{Ba}} \pm 0.29$ \\
\hline Formula (2) & $0.47^{\mathrm{Ca}} \pm 0.31$ & $0.43^{\mathrm{Cab}} \pm 0.33$ & $0.39^{\mathrm{Cb}} \pm 0.40$ & $0.35^{\mathrm{Cbc}} \pm 0.05$ & $0.30^{\mathrm{Cc}} \pm 0.08$ \\
\hline \multirow[t]{2}{*}{ Formula (3) } & $0.40^{\mathrm{Da}} \pm 0.17$ & $0.36^{\mathrm{Dab}} \pm 0.28$ & $0.32^{\mathrm{Db}} \pm 0.25$ & $0.28^{\mathrm{Dbc}} \pm 0.18$ & $0.24^{\mathrm{Dc}} \pm 0.12$ \\
\hline & \multicolumn{5}{|c|}{ pH value } \\
\hline Control & $5.68^{\mathrm{Ac}} \pm 0.20$ & $5.73^{\mathrm{Ab}} \pm 0.22$ & $5.80^{\mathrm{Ab}} \pm 0.11$ & $5.91^{\mathrm{Aa}} \pm 0.13$ & $5.00^{\mathrm{Dd}} \pm 0.17$ \\
\hline Formula (1) & $5.68^{\mathrm{Aa}} \pm 0.19$ & $5.65^{\mathrm{Aab}} \pm 0.24$ & $5.62^{\mathrm{Ab}} \pm 0.10$ & $5.60^{\mathrm{Ab}} \pm 0.32$ & $5.58^{\mathrm{Abc}} \pm 0.26$ \\
\hline Formula (2) & $5.68^{\mathrm{Aa}} \pm 0.17$ & $5.63^{\mathrm{Bab}} \pm 0.19$ & $5.59^{\mathrm{Bb}} \pm 0.21$ & $5.57^{\mathrm{Bb}} \pm 0.13$ & $5.55^{\mathrm{Bbc}} \pm 0.11$ \\
\hline Formula (3) & $5.68^{\mathrm{Aa}} \pm 0.09$ & $5.60^{\mathrm{Cb}} \pm 0.07$ & $5.58^{\mathrm{Cb}} \pm 0.09$ & $5.56^{\mathrm{Cbc}} \pm 0.03$ & $5.54^{\mathrm{Bbc}} \pm 0.07$ \\
\hline
\end{tabular}

Table.5 Microbiological quality of camburger formulated with lemongrass oil storage at $4 \pm 1^{\circ} \mathrm{C}$ for 12 days

\begin{tabular}{|c|c|c|c|c|c|}
\hline Treatments & Zero time & 3 days & 6 days & 9 days & 12 days \\
\hline & \multicolumn{5}{|c|}{ Total Bacterial count as $\log _{10} \mathrm{CFU} / \mathrm{g}$} \\
\hline Control & $1.82^{\mathrm{Ad}} \pm 0.17$ & $2.54^{\mathrm{Ac}} \pm 0.21$ & $2.97^{\mathrm{Ac}} \pm 0.14$ & $3.78^{\mathrm{Ab}} \pm 0.12$ & $4.63^{\mathrm{Aa}} \pm 0.10$ \\
\hline Formula (1) & $1.16^{\mathrm{Bd}} \pm 0.18$ & $1.31^{\mathrm{Bc}} \pm 0.25$ & $1.41^{\mathrm{Bc}} \pm 0.07$ & $1.61^{\mathrm{Bb}} \pm 0.012$ & $1.82^{\mathrm{Ba}} \pm 0.14$ \\
\hline Formula (2) & $1.03^{\mathrm{Bd}} \pm 0.24$ & $1.19^{\mathrm{Bc}} \pm 0.21$ & $1.28^{\mathrm{Bc}} \pm 0.16$ & $1.40^{\mathrm{Bb}} \pm 0.15$ & $1.64^{\mathrm{Ba}} \pm 0.16$ \\
\hline \multirow[t]{2}{*}{ Formula (3) } & $0.76^{\mathrm{Cd}} \pm 0.12$ & $0.92^{\mathrm{Cc}} \pm 0.13$ & $1.12^{\mathrm{Cc}} \pm 0.19$ & $1.25^{\mathrm{Cb}} \pm 0.20$ & $1.49^{\mathrm{Ca}} \pm 0.18$ \\
\hline & \multicolumn{5}{|c|}{ Total Psychrophilic bacteria count as $\log _{10}$ CFU/g } \\
\hline Control & $2.57^{\mathrm{Ad}} \pm 0.10$ & $3.41^{\mathrm{Ac}} \pm 0.15$ & $4.86^{\mathrm{Ab}} \pm 0.11$ & $5.27^{\mathrm{Aa}} \pm 0.16$ & $5.64^{\mathrm{Aa}} \pm 0.14$ \\
\hline Formula (1) & $2.43^{\mathrm{Ac}} \pm 0.12$ & $2.55^{\mathrm{Bb}} \pm 0.20$ & $2.70^{\mathrm{Bb}} \pm 0.23$ & $3.10^{\mathrm{Ba}} \pm 0.09$ & $3.35^{\mathrm{Ba}} \pm 0.11$ \\
\hline Formula (2) & $2.31^{\mathrm{Bc}} \pm 0.14$ & $2.47^{\mathrm{Bb}} \pm 0.18$ & $2.53^{\mathrm{Bb}} \pm 0.19$ & $2.74^{\mathrm{Ba}} \pm 0.07$ & $3.06^{\mathrm{Ba}} \pm 0.15$ \\
\hline Formula (3) & $2.26^{\mathrm{Bc}} \pm 0.16$ & $2.34^{\mathrm{Bb}} \pm 0.13$ & $2.45^{\mathrm{Bb}} \pm 0.08$ & $2.60^{\mathrm{Ba}} \pm 0.06$ & $2.91^{\mathrm{Ba}} \pm 0.12$ \\
\hline
\end{tabular}

Table.6 Sensory evaluation of cameburger formulated with lemongrass oil

\begin{tabular}{|l|c|c|c|c|c|}
\hline \multicolumn{1}{|c|}{ Treatments } & Aroma & Taste & Color & Texture & $\begin{array}{c}\text { Overall } \\
\text { acceptability }\end{array}$ \\
\hline Control & $7.2^{\mathrm{c}} \pm 0.11$ & $7.9^{\mathrm{c}} \pm 0.24$ & $7.7^{\mathrm{c}} \pm 0.17$ & $7.4^{\mathrm{c}} \pm 0.14$ & 30.2 \\
\hline Formula (1) & $8.7^{\mathrm{b}} \pm 0.15$ & $8.8^{\mathrm{b}} \pm 0.19$ & $8.5^{\mathrm{b}} \pm 0.16$ & $8.3^{\mathrm{b}} \pm 0.11$ & 34.3 \\
\hline Formula (2) & $9.2^{\mathrm{a}} \pm 0.18$ & $9.0^{\mathrm{ab}} \pm 0.07$ & $8.7^{\mathrm{ab}} \pm 0.06$ & $8.6^{\mathrm{a}} \pm 0.09$ & 35.5 \\
\hline Formula (3) & $9.5^{\mathrm{a}} \pm 0.13$ & $9.4^{\mathrm{a}} \pm 0.10$ & $9.1^{\mathrm{a}} \pm 0.08$ & $9.0^{\mathrm{a}} \pm 0.12$ & 37 \\
\hline LSD $_{0.05}$ & 0.79 & 0.58 & 0.57 & 0.69 & \\
\hline
\end{tabular}

${ }^{\mathrm{a}-\mathrm{c}}$ means with different superscripts within the same column for each parameter are significantly $(\mathrm{P}<0.05)$ different. 
Control group had the highest TBA value than the other formulated samples. TBA value of both control and camel burger formulated with $0.5 \%$ lemongrass was significantly increased as the time of cold storage increased while, TBA value of cambuger formulated with 0.75 and/ or $1 \%$ lemongrass oil was significantly decreased as the time of storage increased. These results are coincided with (Olorunsanya et al., 2010; Ibrahim and Abu Salem, 2013) they reported that using lemongrass oil with chicken patties as antioxidant were effective and had lower TBARS values than the control samples during refrigerator storage at $4^{\circ} \mathrm{C}$ for 9 days.

No significant differences were found in $\mathrm{pH}$ values for both control and formulated samples at zero time. These results are agreed with Ibrahim \& Abu Salem (2013) they reported that the control and tested chicken patties had the same $\mathrm{pH}$ value at zero time. $\mathrm{pH}$ value of control camebuger was gradually increased during 3, 6 and 9 days and decreased at the end of storage period. The increase in $\mathrm{pH}$ may be due to the accumulation of metabolites by bacterial action in meat and deamination of proteins (Jay, 1996). The $\mathrm{pH}$ of camburger formulated with different levels of lemongrass was significantly decreased as the time of storage increased. This may be due to the lemongrass are a rich source of citrus which possessed an acidity taste and caused decreasing in the $\mathrm{pH}$ value compared with control (Hussein et al., 2015).

Table 5 showed the microbiological quality of camburger formulated with lemongrass oil and storage at $4 \pm 1{ }^{\circ} \mathrm{C}$ for 12 days. Total bacterial count was higher in control sample and no significant differences were found in camburger formulated with 0.5 and/or $0.75 \%$ lemongrass oil. The lowest bacterial count was found cambureger with \% lemongrass. These results are close to that obtained by
Hussein et al., (2015) they found that the control beef burger showed the highest of total bacterial count. While, sample formulated with $2 \%$ lemongrass showed the lowest total bacterial count. During cold storage, the total bacterial count of both control and formulated cambuger was significantly increased, but no significant different was found after 3 and 6 days of storage and such increase was continued as the time of cold storage increased. However, at any time of cold storage cambuger formulated with $1 \%$ lemongrass showed the lowest total bacterial count than the other samples. These results are in line with Ibrahim \&Abu Salem (2013) they found that a remarkable increase was noticed in aerobic plate count of chicken patties throughout cold storage, especially in the control sample at the $6^{\text {th }}$ and $9^{\text {th }}$ days.

From the same table, it can be found that at zero time, control and camburger formulated with $0.5 \%$ lemongrass oil had the higher psychrophilic bacteria followed by camburger formulated with 0.75 and/ or $1 \%$. During cold storage psychrophilic bacteria were significantly increased as the time of storage increased. At any time of cold storage camburger formulated with $1 \%$ lemongrass had the lower psychrophilic bacteria count? This reducing may be due the antimicrobial activity for lemongrass. These results are confirmed by Akin-Osanaiye et al., (2007) and Yaqub Khan et al., (2013).

Table 6 represented the sensory evaluation of camburger formulated with different levels of lemongrass. Generally, camburger formulated with lemongrass oil recorded higher score than control sample. Also, It can be noticed that camburger formulated with $1 \%$ lemongrass recorded the highest score for aroma, taste, color, texture and overall acceptability while, the control burger had the lowest score. These results are close to that 
obtained by Hussein et al., (2015) they found that that the formula made from $2 \%$ lemongrass gave the best acceptability followed by the formula contained equal weight $(1 \%)$ of both lemongrass and thyme.

Addition of lemongrass oil to camel burger decreased the cooking loss $\%$, reduction in diameter $\%$, the total bacterial count, psychrophilic bacteria, TBA, TVBN value during cold storage for 12 days at $4^{\circ} \mathrm{C}$ and improved the sensory evaluation of the product. It could be concluded that lemongrass oil can be successfully use as natural antioxidant and antimicrobial agent in meat products.

\section{References}

A.M.S.A. 1995. American Meat Science Association. Research guidelines for cookery, sensory evaluation and instrumental tenderness measurements of fresh beef. Chicago, IL, USA.

Adams, R. 2007. Identification of essential oil components by gas chromatography/mass spectrometry, Allured Publishing Carol Steam Ilionois, USA.

Akin-Osanaiye, B.C., Agbaji, A.S. and Dakare, 2007. Antimicrobial activity of oils and extracts of Cymbopogon citrates (Lemongrass), Eucalyptus citriodora and Eucalyptus camaldulensis. J. Med. Sci., 7(4):694697.

Armitage, P and Berry, G. 1987. Statistical Method in Medical Research. Blackwell, Oxford, UK, PP: 93-213.

Barbosa, L. C. A., Pereira, U. A., Martinazzo, A. P., Maltha, C. R.Á., Teixeira, R. R., and Melo, E. C. 2008. Evaluation of the Chemical Composition of Brazilian Commercial Cymbopogon citratus (D.C.) Stapf Samples. Molecules, (13) 1864-1874.
Berry, B.W. 1993. Fat level and freezing temperature affect sensory, shear cooking and composition properties of ground beef patties. Journal of Food Science, 58 (1):34-42.

Bozin B, Mimica-Dukic N, Samojlik I, Jovin E. 2007. Antimicrobial and antioxidant properties of rosemary and sage (Rosmarinus officinalis L. and Salvia officinalis L., Lamiaceae) essential oils. J Agric Food Chem., 55: (19) 78797885.

Byrd, S.J., 2001. Using antioxidants to increase shelf life of food products. Cereal Foods World, 46: 48-53.

Cheel J, Theoduloz C, Rodríguez J, SchmedaHirschmann G 2005. Free radical scavengers and antioxidants from Lemongrass (Cymbopogon citratus (DC.) Stapf.). J. Agric. Food Chem. 53: 2511-2517.

Defreitas, Z., J. G. Sebranek, D. G. Olson, and J. M. Carr. 1997. Freeze/thaw stability of cooked pork sausages as affected by salt, phosphate, $\mathrm{pH}$ and carrageenan. J. Food Sci., 62(3):551554.

Hussein, S. A., Shahin, MF. S.A., and Masoud, M.R.M. 2015. Effect of using lemongrass and thyme on some beefburger characteristics. Egypt. J. Agric. Res., 93 (1), 133-144.

Ibrahim, H. M., Ferial M. Abu Salem 2013. Effect of Adding Lemongrass and Lime Peel Extracts on Chicken Patties Quality. Journal of Applied Sciences Research, 9(8): 5035-5047.

ISO4833. 1991. Microbiology. General Guidance for the Enumeration of Microorganisms Colony-Count Technique at $30^{\circ} \mathrm{C}$.

Jay, J. M. 1972. Mechanism and detection of microbial spoilage in meats at low temperatures: A status report. J. Milk Food Tech., 35 (8): 467-471.

Johnston, J.E., Sepe, H. A., Miano, C.L., 
Brannan, R.G., Alderton, A.L. 2005. Honey inhibits lipid oxidation in readyto eat ground beef patties. Meat Sci., 70: 627- 631 .

Khadri, A., M. Neffati, S. Smiti, P. Falé, A.R.L. Lino, M.L.M. Serralheiro, M.E.M. Araùjo, 2010. Antioxidant, antiacetylcholine sterase and antimicrobial activities of Cymbopogon schoenanthus L. Spreng (lemon grass) from Tunisia. LWT - J. Food Sci. Technol., 43: 331-336.

Kirk, R.S and Sawyer, R. 1991. Pearson's Composition and Analysis of Foods. $9^{\text {th }}$ Ed., AWL, Harlow.

Mailgaad, M., Civille, G. V., \& Carr, B. T. 1999. Sensory evaluation techniques. Boca Raton, FL: CRS Press.

Matasyoh, J. C., Wagara, I. N., Nakavuma, J. L., and Kiburai, A. M. 2011. Chemical composition of Cymbopogon citrates essential oil and its effect on mycotoxigenic Aspergillus species. African Journal of Food Science Vol. 5(3), pp. 138-142.

Olorunsanya, A.O., Olorunsanya, E.O., Bolu, S.A.O., Adejumobi, C.T., and Kayode, R.M.O. 2010. Effect of graded levels of lemongrass (Cymbopogon citratus) on oxidative stability of raw or cooked pork patties. Pakistan Journal of
Nutrition 9 (5): 467-470.

Portuguese Standard 2307. 1987. Food Microbiology General Guidance for the Enumeration of psychrotrophic microorganisms.

Rosmini, M. R., Perlo, F., Perez-Alvarez, J. A., Pagan-Moreno, M. J., Gago-Gago, M. A., Lopez-Santovea, F., 1996. TBA test by extractive method applied to pate. Meat Science, 42, 103-110.

Vilelaa, J.; Martins, D.; Monteiro-Silva, F.; González-Aguilar, G.; and Saraiva, C. 2016. Antimicrobial effect of essential oils of Laurus nobilis $L$. and Rosmarinus officinallis $L$. on shelf-life of minced "Maronesa" beef stored under different packaging conditions. Food Packaging and Shelf Life. 8, 7180.

Wierbicki, E. and Deatherage, F. E. 1958. Determination of water holding capacity of fresh meats. Agric. Food Chem., 6 (5): 387-392.

Yaqub, K. M., Gupta, P., Singh, VK. Yadav, S. and Verma, V. K. 2013. Cymbopogon Citrates Oil Showing Antimicrobial Activity against Microbes of Environmental, Clinical and Food Origin. Asian J. Pharm. Tech. (3): 2, 67-72.

\section{How to cite this article:}

Engy F. Zaki, Abdel Aziz Nadir, Ibrahim Mohamed Fouad Helmy and Nahed M. Abdel Maguid. 2018. Antioxidant and Antimicrobial Effects of Lemongrass (Cymbopogon citrates) Oil on the Quality Characteristics of Camel Burger "Camburger" under Refrigerated Storage. Int.J.Curr.Microbiol.App.Sci. 7(03): 3623-3631. doi: https://doi.org/10.20546/ijcmas.2018.703.418 